\title{
Dust Trails of 8P/Tuttle and the Unusual Outbursts of the Ursid Shower
}

\author{
P. Jenniskens \\ SETI Institute, NASA/Ames Research Center, Mail-Stop 239-4, Moffett Field, California 94035-1000 \\ E-mail: pjenniskens@mail.arc.nasa.gov \\ E. Lyytinen \\ Kehäkukantie 3 B, 00720 Helsinki, Finland
}

M. C. de Lignie, C. Johannink, K. Jobse, R. Schievink, and M. Langbroek

Dutch Meteor Society, Lederkarper 4, 2318 NB Leiden, The Netherlands

M. Koop, P. Gural, and M. A. Wilson

California Meteor Society, 1037 Wunderlich Drive, San José, California 95129-3159

and

I. Yrjölä, K. Suzuki, H. Ogawa, and P. de Groote

Global-MS-Net, Jukolantie 16, FIN-45740, Kuusankoski, Finland

Received June 26, 2001; revised February 2, 2002

We calculate the position of dust trails from comet $8 \mathrm{P} /$ Tuttle, in an effort to explain unusual Ursid meteor shower outbursts that were seen when the comet was near aphelion. Comet 8P/Tuttle is a Halley-type comet in a 13.6-year orbit, passing just outside of Earth's orbit. We find that the meteoroids tend to be trapped in the 12:14 mean motion resonance with Jupiter, while the comet librates in a slightly shorter period orbit around the 13:15 resonance. It takes 6 centuries to decrease the perihelion of the meteoroid orbits enough to intersect Earth's orbit, during which time the meteoroids and comet separate in mean anomaly by 6 years, thus explaining the 6-year lag between the comet's return and Ursid outbursts. The resonances also prevent dispersion along the comet orbit and limit viewing to only one year in each return. We identified past dust trail encounters with dust trails from 1392 (Dec. 1945) and 1378 (Dec. 1986) and predicted another outburst on 2000 December 22 at around 7:29 and 8:35 UT, respectively, from dust trails dating to the 1405 and 1392 returns. This event was observed from California using video and photographic techniques. At the same time, five Global-MS-Net stations in Finland, Japan, and Belgium counted meteors using forward meteor scatter. The outburst peaked at 8:06 \pm 07 UT, December 22, at zenith hourly rate $\sim \mathbf{9 0}$ per hour, and the Ursid rates were above half peak intensity during $4.2 \mathrm{~h}$. We find that most Ursid orbits do scatter around the anticipated positions, confirming the link with comet 8P/Tuttle and the epoch of ejection. The 1405 and 1392 dust trails appear to have contributed similar amounts to the activity profile. Some orbits provide a hint of much older debris being present as well. This work is the strongest evidence yet for the relevance of mean motion resonances in Halley-type comet dust trail evolution. (c) 2002 Elsevier Science (USA)

\section{INTRODUCTION}

When planetary perturbations put narrow comet dust trails in Earth's path, brief showers called meteor outbursts are observed (Kresák 1993, Williams and Wu 1994, Jenniskens 1995, 1997, Jenniskens et al. 1997). Calculating the perturbations on test particles released at perihelion has made it possible to identify many past Leonid storms with the ejecta from specific perihelion returns of the parent comet 1-9 revolutions ago. The recent Leonid meteor storm of 1999 November 18 and a second outburst half a day later were caused by ejecta from Comet 55P/Tempel-Tuttle released at the returns of 1899 and 1866, respectively (Kondrat'eva and Reznikov 1985, Lyytinen 1999, McNaught and Asher 1999). And three calculated encounters with dust from the 1932, 1733, and 1866 returns were observed much as predicted (Jenniskens 2001a). Similar identifications for short-period comets have been made for outbursts of the June Bootids, Beilids, and Draconids (Reznikov 1983). The work to date serves as a framework for comprehensive models that can correctly predict the time, peak activity, duration, and particle size distribution of the meteor outbursts. 
To understand the manifestation of dust trails as meteor showers, one of the questions that need to be addressed is the role of mean motion resonances in maintaining cohesion of the dust trails. Asher et al. (1999) pointed out that dust trails can maintain their character for a very long time when the particles are trapped in mean-motion resonances. When planetary perturbations change the orbital period of the particles, they catch up on each other and disperse into a wider component over time, which has been called the shower "filament" (Jenniskens and Betlem 2000). More so, close encounters with the planets tend to scatter the grains into an annual shower component. While (fragments of) young dust trails may escape close encounters with Jupiter, older trails tend to maintain their cohesion only if the meteoroids continue to avoid dynamically unfavorable conditions. Because of this, outbursts of Halley-type comets are typically observed when the comet is nearby, the comet itself being trapped in such mean motion resonance (Jenniskens 1995).

During a cold winter night in 1945, visual observers at Skalnaté Pleso Observatory witnessed an outburst of meteors radiating from Ursa Minor (Becvár 1946). Ceplecha (1951) calculated orbital elements from three single-station trajectories and an assumed velocity and found good general agreement with the orbit of Comet 8P/Tuttle. This confirmed an earlier notion by W. F. Denning, who had observed a minor annual meteor shower in Ursa Minor (Denning 1912), but the weak nature of the shower prompted him to add that such a relationship "may be an accidental accordance" (Denning 1921). It took the outburst in 1945 to put this shower on the map. Years later, Norwegian observers Kai Gaarder and Lars Trygve-Heen saw a similarly spectacular outpouring of Ursid meteors over a period of $3 \mathrm{~h}$ in December of 1986 (Jenniskens 1987).

Comet 8P/Tuttle has Tisserand parameter 1.6, which makes it a Halley-type comet, despite its relatively short orbital period of about 13.6 years. However, Ursid outbursts seem to differ from the Leonid outbursts of 55P/Tempel-Tuttle in two ways: they occur when the comet is near aphelion rather than near perihelion, and they occur despite the comet orbit being over six times further from Earth's orbit. During its most recent return in 1994, 8P/Tuttle crossed the ecliptic plane +0.061 AU outside of Earth's orbit (Marsden and Williams 1994).

Comet 8P/Tuttle was about to be back at aphelion in March of 2001 when we considered the possibility of another Ursid outburst in December of 2000. Application of the Leonid showerprediction models to the Ursids in early December revealed that Earth was about to cross the dust trails of 1405 and 1392 on December 22, 2000 (see below). If confirmed, this would be only the second time that meteors had been traced to a specific epoch of ejection from Halley-type comets (following the Leonid predictions), extending the range of validity back in time. The prediction was summarized in the December 2000 issue of WGN, the Journal of the IMO (International Meteor Organization), a preprint of which was widely circulated on the internet and in the astronomical community prior to the shower (Jenniskens and Lyytinen 2000). An announcement was made as well in the IAU
Circulars (Jenniskens 2000a). In order to confirm the forecast, video and photographic observations were deployed in California, while radio forward meteor-scatter observers worldwide gathered meteor counts. Early reports confirmed that an outburst occurred around the predicted time at the anticipated level of activity (Jenniskens 2000b, Jenniskens and Lyytinen 2001).

This paper elaborates on these early papers by comparing the results of the observations to the predictions that were made. New results from the video observations are presented. We discuss the role of mean motion resonances in the orbital evolution of the Ursid dust trails 44 and 45 revolutions after ejection from the comet and conclude that the observations may show a more complex scenario than originally envisioned.

\section{PRIOR OBSERVATIONS}

\section{The Ursid Filament}

Let us first examine other manifestations of the Ursid shower in recent years, to show the unusual aspects of these aphelion outbursts. The annual Ursid shower has a peak zenith hourly rate $(Z H R)$ of $=8 \mathrm{~h}^{-1}$ and has a $Z H R>1 \mathrm{~h}^{-1}$ from about December 18 to 25 (Jenniskens 1994). Planetary perturbations have dispersed this dust sufficiently widely and all along the comet orbit for annual encounters with Earth. This component represents the level of Ursid activity in such quiet years as 1998 and 1999.

On top of that, there are frequent outbursts when the comet is near perihelion. Figure 1 shows the rate of meteors detected by forward meteor-scatter above the normal daily activity from December 19 to 24. Shortly before the comet's return in December 1993, Bob Lunsford of Mt. Laguna, California, observed the ascending branch of an outburst rising to $Z H R_{\max }=$ $100 \mathrm{~h}^{-1}$. The meteors were of average brightness, with a magnitude distribution index $r=N(m+1) / N(m)=2.5$. Japanese observer H. Shioi observed a similar enhanced rate one year later (Ohtsuka et al. 1995), from which we have $Z H R_{\max }=50 \mathrm{~h}^{-1}$ $(r=2.6)$. After that, rates gradually declined. In 1996, rates were still elevated at $Z H R_{\max }=25 \mathrm{~h}^{-1}$ (Langbroek 1997), and of order $Z H R_{\max }=16 \mathrm{~h}^{-1}$ in 1997 (this work). No significant enhanced activity over the normal annual shower rates occurred in 1999. Moreover, similar outbursts were observed during the previous return of the comet at perihelion, by Japanese observers in 1981 (Ohtsuka 1994) and by Jos Nijland and Hans Breukers of the Dutch Meteor Society in 1982, who observed the descending branch starting at $Z H R \sim 35 \mathrm{~h}^{-1}$ (this work, from DMS archive data).

These perihelion outbursts occurred at a significantly different point in the orbit than the 1986 outburst, about $6 \mathrm{~h}$ earlier, and were significantly wider. If the zenith hourly rate has the usual functional form (Jenniskens 1994),

$$
Z H R \sim 10^{-B\left|\lambda_{0}-\lambda_{\mathrm{o}}^{\max }\right|}
$$

with solar longitude $\lambda_{\mathrm{o}}$ a measure of time, we find $B=2.5 \pm$ 

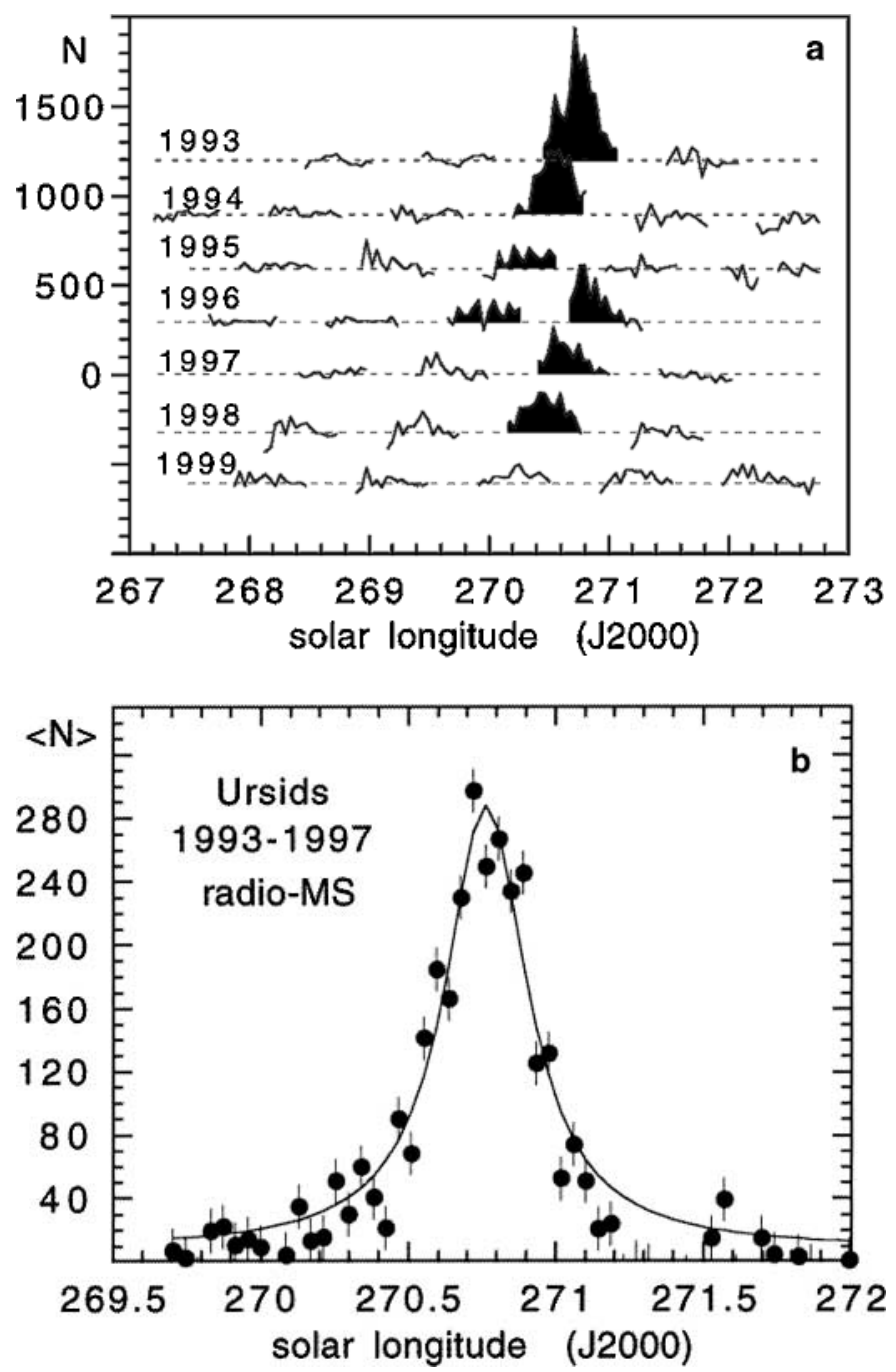

FIG. 1. (a) Ursid Filament as observed around the 1994 perihelion passage of Comet 8P/Tuttle by the forward meteor-scatter technique. Hourly counts $(N)$ are raw reflections after subtraction of the daily background rate of sporadic meteors. No correction for observability has been applied. (b) The mean activity profile of the 1993-1997 Ursid outbursts derived from data shown in Fig. 1a, after correction for observability.

0.5 degree $^{-1}$ from the data in Fig. 1. On the other hand, if the function form is a Lorentz profile, as in the case of the 1999 Leonid meteor storm (Jenniskens et al. 2000a),

$$
Z H R \sim \frac{(W / 2)^{2}}{\left(\lambda_{\mathrm{o}}-\lambda_{\mathrm{o}}^{\max }\right)^{2}+(W / 2)^{2}}
$$

then we find a Lorenz curve full-width-at-half-maximum $W=$ $0.35 \pm 0.05^{\circ}$. The Lorentzian curve adds a tail to the exponential part described by Eq. (1). In any case, the shower is much wider than past Leonid and Draconid storms, which have a fullwidth-at-half-maximum about a factor of 10 smaller (Jenniskens 1995). Instead, the width is similar to the "filament" that was responsible for the Leonid outbursts of 1994-1998 (Jenniskens

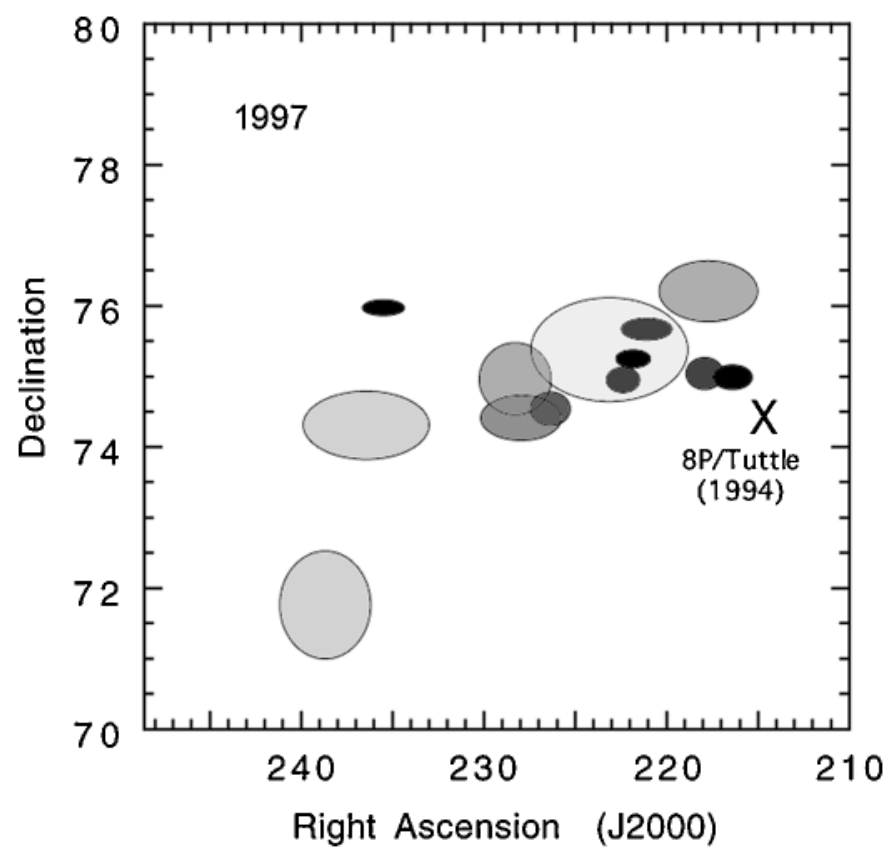

FIG. 2. Radiant positions of 1997 Ursids (Table I). The size of the ovals and the corresponding density of shading are proportional to the accuracy of the measurement. An " $\mathrm{X}$ " shows the theoretical radiant position derived from the 1994 comet orbit, after $q$ was changed to intersect Earth's orbit.

and Betlem 2000) and the Perseid outbursts of 1989-1997 (Jenniskens et al. 1998).

These activity profiles describe the nodal distribution of the orbits and dispersion of dust along the comet orbit. The only other information on the dynamical properties of this dust is a series of 13 outburst Ursid orbits obtained from two-station video observations in California over a period of $4 \mathrm{~h}$ in December

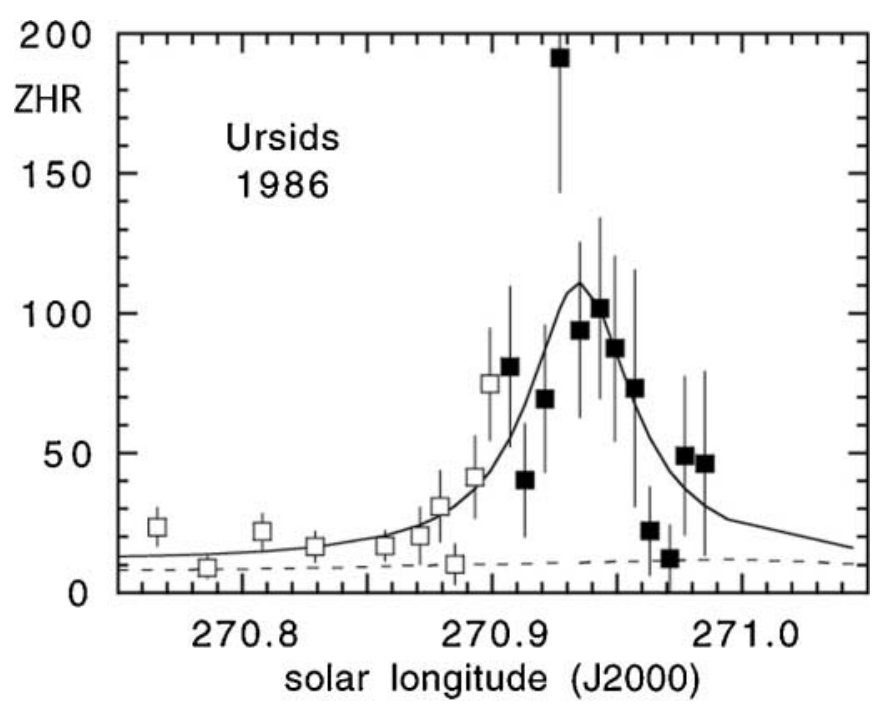

FIG. 3. Activity curve for the Ursid outburst in 1986. ZHR is zenith hourly rate. Open squares are results from Ursid counts by observer Kai Gaarder and filled squares are results from Lars Trygve-Heen (Jenniskens 1995). 
TABLE I

Orbital Elements of Ursid Meteors (J2000)

\begin{tabular}{|c|c|c|c|c|c|c|c|c|c|c|c|c|c|c|c|c|c|c|}
\hline Code & Node & Mv & $q$ & \pm & $1 / a$ & \pm & I & \pm & $w$ & \pm & $V \mathrm{~g}$ & \pm & $R A$ geo & \pm & $D E C$ geo & \pm & $H \mathrm{~b}$ & $\mathrm{He}$ \\
\hline \multicolumn{19}{|c|}{1997} \\
\hline 97600 & 270.5500 & 3 & 0.937 & 0.002 & 0.20 & 0.03 & 53.8 & 0.4 & 206.6 & 0.6 & 33.6 & 0.4 & 216.4 & 1.1 & 75.0 & 0.2 & 107.4 & 94.8 \\
\hline 97603 & 270.5600 & 5 & 0.952 & 0.003 & 0.20 & 0.05 & 51.5 & 0.9 & 201.8 & 1.0 & 32.3 & 0.8 & 228.3 & 2.0 & 75.0 & 0.5 & 105.7 & 99.7 \\
\hline 97605 & 270.6000 & 3 & 0.951 & 0.002 & 0.27 & 0.06 & 51.0 & 1.1 & 202.8 & 0.7 & 31.5 & 1.0 & 226.4 & 1.1 & 74.5 & 0.3 & 104.5 & 94.0 \\
\hline 97610 & 270.6300 & 6 & 0.944 & 0.007 & 0.28 & 0.06 & 50.7 & 1.1 & 205.1 & 2.3 & 31.3 & 0.9 & 223.1 & 4.3 & 75.4 & 0.7 & 103.2 & 99.3 \\
\hline 97611 & 270.6300 & 4 & 0.945 & 0.002 & 0.26 & 0.02 & 51.5 & 0.5 & 204.8 & 0.6 & 31.9 & 0.4 & 222.4 & 0.9 & 74.9 & 0.2 & 107.7 & 93.2 \\
\hline 97613 & 270.6400 & 3 & 0.942 & 0.002 & 0.17 & 0.03 & 52.7 & 0.4 & 204.9 & 0.7 & 33.3 & 0.4 & 221.1 & 1.4 & 75.7 & 0.2 & 105.2 & 92.8 \\
\hline 97618 & 270.6500 & 4 & 0.935 & 0.005 & 0.24 & 0.04 & 51.5 & 0.8 & 207.5 & 1.4 & 32.2 & 0.7 & 217.7 & 2.7 & 76.2 & 0.4 & 103.1 & 95.9 \\
\hline 97619 & 270.7000 & 4 & 0.953 & 0.003 & 0.24 & 0.08 & 51.2 & 1.5 & 201.7 & 1.4 & 31.8 & 1.4 & 227.9 & 2.2 & 74.4 & 0.3 & 101.2 & 94.4 \\
\hline 97622 & 270.7100 & 0 & 0.944 & 0.002 & 0.16 & 0.03 & 53.0 & 0.4 & 204.2 & 0.6 & 33.4 & 0.4 & & 1.0 & 75.3 & 0.1 & 108.4 & 88.3 \\
\hline 97625 & 270.7200 & 2 & 0.939 & 0.002 & 0.20 & 0.03 & 53.4 & 0.5 & 206.1 & 0.6 & 33.4 & 0.4 & 217.9 & 1.0 & 75.0 & 0.2 & 102.8 & 89.6 \\
\hline \multicolumn{19}{|c|}{2000} \\
\hline 20506 & 270.5926 & 2 & 0.949 & 0.004 & 0.16 & 0.04 & 54.0 & 0.6 & 202.5 & 1.5 & 33.9 & 0.4 & 223.2 & 2.8 & 74.1 & 0.5 & 107.0 & 99.4 \\
\hline 20513 & 270.6176 & 4 & 0.939 & 0.002 & 0.15 & 0.05 & 52.1 & 0.8 & & 0.6 & 33.1 & 0.7 & & 1.0 & 76.6 & 0.3 & 103.7 & 94.7 \\
\hline 20515 & 270.6260 & 4 & 0.940 & 0.002 & 0.30 & 0.05 & 51.5 & 0.9 & 206.7 & 0.8 & 31.6 & 0.7 & & 0.9 & 74 & 0.5 & 3.0 & 94.6 \\
\hline 20518 & 270.6391 & 4 & 0.941 & 0.002 & 0.19 & 0.06 & 53.5 & 1.1 & 205.3 & 0.8 & & 0.9 & & & & & & 92.4 \\
\hline 20521 & 270.6455 & 3 & 0.939 & 0.002 & 0.35 & 0.0 & 51 & 1.3 & 207.6 & 1.0 & & 1.0 & & 0.7 & & 0.4 & & 2.5 \\
\hline 20525 & 270.6577 & 2 & 0.946 & 0.001 & 0.30 & 0.12 & 51.3 & 2.5 & 204.8 & 1.5 & 31.5 & 2.1 & 222.3 & 0.4 & 74 & 0.3 & 111.3 & 81.4 \\
\hline 20526 & 270.6610 & 3 & 0.943 & 0.002 & 0.21 & 0.06 & 51.3 & 1.1 & & 0.8 & & 0.9 & & 1.2 & & 0.4 & & 95.6 \\
\hline 20527 & 270.6695 & 5 & 0.945 & 0.002 & 0.13 & 0.03 & 55.2 & 0.5 & 203.9 & 0.6 & 34.8 & 0.4 & & 0. & & .5 & & 96.3 \\
\hline 20528 & 270.6714 & 5 & 0.940 & 0.002 & 0.34 & 0.08 & 50.4 & 1.8 & 207.0 & 1.2 & & & & & & & & 93.3 \\
\hline 20532 & 270.6775 & 3 & 0.941 & 0.003 & 0.24 & 0.14 & 52.0 & 2.6 & 205.9 & 1.7 & & & & 1.1 & & & & 95.3 \\
\hline 20536 & 270.6853 & 4 & 0.934 & 0.002 & 0.23 & 0.06 & 51.4 & 1.1 & 207.9 & 0.8 & 32.2 & 0.9 & 217.1 & 0.9 & 76 & 0.5 & 102.2 & 93.8 \\
\hline 20537 & 270.6872 & 5 & 0.941 & 0.002 & 0.24 & 0.05 & 51 & 0.9 & 205.7 & 0.7 & & 0.7 & & 0. & & .5 & & 95.3 \\
\hline 20539 & 270.6882 & 3 & 0.934 & 0.006 & 0.29 & 0.10 & 51.6 & 1.9 & 208.4 & 2.3 & & & & & & & & 91.5 \\
\hline 20540 & 270.6885 & 4 & 0.933 & 0.002 & 0.17 & 0.05 & 50.5 & 0.9 & 207.5 & 0.7 & & 0.8 & & & & 0.4 & & 95.2 \\
\hline 20542 & 270.6996 & 2 & 0.941 & 0.000 & 0.22 & 0.02 & 52.5 & 0.5 & 205.6 & 0.3 & 32.7 & 0.4 & 219 & 0.0 & & 0.0 & 108.2 & 87.1 \\
\hline 20701 & 270.7212 & 3 & .0946 & 0.001 & 0.22 & 0.03 & 52.8 & 0.5 & 204.2 & 0.4 & 32.9 & 0.5 & 221.7 & 0.6 & & 0.3 & 107.0 & 98.0 \\
\hline 20703 & 270.7447 & 3 & 0.934 & 0.001 & 0.19 & 0.06 & 52.4 & 0.9 & 207 & 0.6 & & 0.8 & & 0. & & & 107.6 & 100.8 \\
\hline 20704 & 270.7460 & 2 & 0.940 & 0.001 & 0.2 & 0.04 & 51 & 0.6 & 205.9 & 0. & & & & & & & & 95.3 \\
\hline 20705 & 270.7478 & 4 & & 0.003 & 0.21 & & 52.4 & 0.4 & & 0.8 & & 0.4 & & 1.7 & & 0.2 & & 100.4 \\
\hline 20706 & 270.7495 & 4 & 0.946 & 0.001 & 0.29 & 0.03 & 51.6 & 0.5 & 204.6 & 0.5 & 31.7 & 0.4 & 222 & 0.9 & 74 & 0.2 & 106.7 & 98.5 \\
\hline 20707 & 270.7591 & 6 & 0.938 & 0.001 & 0.20 & 0.09 & 53.0 & 1.5 & 206.3 & 0.9 & 33.2 & 1.4 & 217 & 0. & & 0.3 & 107.9 & 102.5 \\
\hline 20708 & 27 & 4 & & 0.00 & 0.2 & 0.03 & 51 & 0.4 & & 0.4 & & 0.4 & & 0. & & & & 99.5 \\
\hline 20709 & 0.7609 & 3 & 0.935 & 0.00 & 0.1 & 0.0 & 52 & 0. & 206 & 0. & & & & & 76 & & .7 & 98.1 \\
\hline 20710 & 270.7657 & 5 & & 0.001 & 0.23 & & 52.0 & 0.6 & 203.1 & 0.4 & 32.4 & 0.5 & & 0.7 & & 0.2 & 105.7 & 100.7 \\
\hline 20711 & 270.7662 & 5 & 0.944 & 0.001 & 0.27 & 0.03 & 52.2 & 0.4 & 205.1 & 0.5 & 32.2 & 0.4 & 220.7 & 0.8 & 74 & 0.2 & 106.3 & 97.9 \\
\hline 20712 & 270.7747 & 4 & 0.937 & 0.002 & 0.27 & 0.05 & 51.4 & 0.9 & & 0.7 & & 0.8 & & & & & & 99.9 \\
\hline 20713 & 270.7836 & 5 & 927 & 0.002 & 0.1 & 0.09 & 54.4 & 1.4 & 208.8 & 0.9 & 34.5 & 1.3 & & 1. & 7. & & .1 & 99.8 \\
\hline 20714 & 0.783 & 4 & 0.943 & 0.002 & 0.1 & 0.03 & 52.4 & 0.4 & 204.4 & 0.5 & 33 & 0.4 & & 1. & 76 & 0.2 & 107 & 98.9 \\
\hline 20715 & 270.7879 & 5 & & 0.001 & 0.21 & 0.04 & 52.2 & 0.7 & 205.4 & 0.5 & 32.7 & 0.6 & & 0.7 & & 0.3 & 106.7 & 99.8 \\
\hline 20716 & 270.7907 & 4 & 0.924 & 0.002 & 0.14 & 0.04 & 54.4 & 0.6 & 209.7 & 0.6 & 34.5 & 0.6 & 209.4 & 1.1 & 76 & 0.2 & 108.1 & 97.6 \\
\hline 20717 & 707918 & 4 & 0.934 & 0.002 & 0.28 & 0.03 & 52.0 & 0.5 & & 0.6 & & 0.4 & & 0.9 & & & & 100.3 \\
\hline 20718 & 270.7919 & 1 & 0.942 & 0.001 & 0.2 & 0.03 & 53 & 0. & 205 & 0.5 & 33.0 & 0.4 & & 0. & 74 & & 111 & 92.7 \\
\hline 20719 & 0.8081 & 4 & & 0.00 & 0. & 0. & 53 & 1. & 206 & 1.0 & & 1.6 & & 0 & 75 & 0.2 & & 96.9 \\
\hline 20720 & 270.8089 & 4 & 0.929 & 0.002 & 0.13 & 0.05 & 54.1 & 0.8 & 208.3 & 0.6 & 34.4 & 0.7 & 212.7 & 1.0 & 76.2 & 0.2 & 105.4 & 97.8 \\
\hline 20721 & 270.8182 & 2 & 0.947 & 0.001 & 0.19 & 0.03 & 52.9 & 0.4 & 203.4 & 0.5 & 33.1 & 0.4 & 223.0 & 0.9 & 74.6 & 0.2 & 105.4 & 99.2 \\
\hline 20722 & & 4 & & 0.001 & 0.13 & 0.10 & 54.1 & 1.6 & & 0.9 & & 1.5 & & 0.9 & & & & 98.2 \\
\hline 20723 & م 728 & 4 & 0.940 & 0.001 & 0.3 & 0.0 & 51. & 0.6 & 207.0 & 0.6 & 31. & 0. & & 0. & 74 & 0.2 & 105 & 99.0 \\
\hline 20725 & 2 & 3 & & & & & & & & 0.5 & & 0.4 & & 0.9 & & 0.2 & & 95.8 \\
\hline 20726 & 270.7622 & 3 & 0.943 & 0.002 & 0.17 & 0.06 & 53.2 & 0.9 & 204.6 & 0.6 & 33.5 & 0.8 & 220.9 & 1.0 & 75.2 & 0.2 & 107.9 & 97.0 \\
\hline 20727 & 270.7643 & 4 & 0.941 & 0.001 & 0.22 & 0.03 & 53.0 & 0.4 & 205.5 & 0.5 & 33.0 & 0.4 & 219.3 & 0.8 & 74 & 0.2 & 106.8 & 97.6 \\
\hline 20728 & 270.7648 & 4 & & 0.002 & 0.1 & 0.0 & 52. & 0. & 207.1 & 0.5 & 33.1 & 0.5 & & & 15 & & 108.0 & 97.0 \\
\hline 20739 & 70.7717 & 1 & & & 0.1 & 0.0 & 53. & 0 . & & 0.5 & & 0.4 & & 1. & 76 & .2 & & 95.0 \\
\hline 20730 & $0.1 / 40$ & 3 & & & & & & & & 0.8 & & 0.7 & & & & 0.2 & & 99.3 \\
\hline 20731 & 270.7776 & 1 & 0.938 & 0.001 & 0.18 & 0.03 & 53.2 & 0.4 & 206.1 & 0.5 & 33.5 & 0.4 & 218.0 & 0.9 & 75.5 & 0.2 & 111.3 & 91.9 \\
\hline 20733 & 270.7904 & 4 & 0.946 & 0.001 & 0.19 & 0.03 & 52.1 & 0.5 & 204.0 & 0.5 & 32.7 & 0.4 & 223.5 & 0.9 & 75.3 & 0.2 & 107.1 & 98.0 \\
\hline 20734 & 270.7928 & 4 & 0.944 & 0.001 & 0.22 & 0.06 & 52.8 & 1.1 & 204.7 & 0.7 & 32.9 & 1.0 & 220.8 & 0.9 & 74.6 & 0.2 & 105.8 & 98.1 \\
\hline 20735 & 270.7955 & 4 & 0.922 & 0.003 & 0.22 & 0.03 & 53.5 & 0.4 & 210.8 & 0.8 & 33.5 & 0.4 & 208.7 & 1.5 & 75.8 & 0.2 & 106.8 & 100.0 \\
\hline
\end{tabular}


TABLE I-Continued

\begin{tabular}{ccccccccccccccccccc}
\hline Code & Node & $\mathrm{Mv}$ & $q$ & \pm & $1 / a$ & \pm & $\mathrm{I}$ & \pm & $w$ & \pm & $V \mathrm{~g}$ & \pm & $R$ Ageo & \pm & $D E C$ geo & \pm & $H \mathrm{~b}$ & $H \mathrm{e}$ \\
\hline 20736 & 270.8068 & 2 & 0.941 & 0.002 & 0.21 & 0.03 & 52.1 & 0.4 & 205.7 & 0.5 & 32.7 & 0.4 & 220.4 & 1.0 & 75.6 & 0.2 & 110.6 & 93.0 \\
20738 & 270.8092 & 4 & 0.948 & 0.002 & 0.21 & 0.03 & 53.0 & 0.4 & 203.4 & 0.7 & 33.0 & 0.4 & 222.8 & 1.2 & 74.2 & 0.2 & 106.7 & 99.5 \\
20739 & 270.8126 & 2 & 0.937 & 0.002 & 0.19 & 0.03 & 52.4 & 0.4 & 206.7 & 0.6 & 33.0 & 0.4 & 218.1 & 1.0 & 75.9 & 0.2 & 108.8 & 93.8 \\
20740 & 270.8147 & 3 & 0.926 & 0.002 & 0.21 & 0.03 & 53.2 & 0.5 & 209.7 & 0.6 & 33.4 & 0.4 & 211.1 & 1.1 & 75.9 & 0.1 & 106.4 & 95.8 \\
20741 & 270.8196 & 1 & 0.939 & 0.002 & 0.15 & 0.03 & 53.5 & 0.4 & 205.7 & 0.5 & 33.8 & 0.4 & 218.4 & 1.0 & 75.5 & 0.2 & 116.2 & 85.6 \\
20742 & 270.8221 & 2 & 0.931 & 0.002 & 0.20 & 0.03 & 53.1 & 0.4 & 208.4 & 0.6 & 33.3 & 0.4 & 213.9 & 1.1 & 75.9 & 0.2 & 108.2 & 93.5 \\
\hline
\end{tabular}

Note. Mv is the absolute visual magnitude at $D=100 \mathrm{~km} ; q=$ perihelion distance; $a=$ semimajor axis; $i=$ inclination; $w=$ argument of perihelion; $V \mathrm{~g}$ is the geocentric velocity after subtraction of the effects of Earth's gravity; $R A$ and $D E C$ are the geocentric radiant positions corrected for the effects of Earth's gravity, while $H \mathrm{~b}$ and $H \mathrm{e}$ are the beginning and end point of the video trajectories.

of 1997 (Table I-orbits with prefix “97”). Table II lists the mean of the 10 orbits that are most clustered. The radiant of these orbits shows significant scatter with a wide range in right ascension, more than in declination, even without three possible outliers (Fig. 2; the outliers are the three left-most points). The mean orbit differs significantly from that calculated by Ceplecha (1951) for the 1945 Ursid outburst (Table II).

\section{Aphelion Outbursts}

The brief aphelion outbursts of 1945 and 1986 (Fig. 3) happened when the comet was near aphelion and, remarkably, were not repeated the next year in 1946 (Ceplecha 1951), nor in 1987 (Jenniskens 1988c). The 1945 outburst was 6.089 years after perihelion passage in 1939, Nov. 10.6, while the 1986 outburst happened 6.002 years after the 1980 , Dec. 14.7 perihelion passage. Compare this to the anticipated encounter in 2000, which was 6.549 years after the 1994 , June 25.3 passage of the parent comet.

These aphelion outbursts are much more narrow than the perihelion outbursts discussed in the previous section, by a factor of 6 , with $B=17 \pm 3$ degree $^{-1}$ rather than $B=2.5$ degree $^{-1}$, or a Lorentz width of $W=0.05 \pm 0.01^{\circ}$. Note the difference in scale between Figs. $1 \mathrm{~b}$ and 3. Also, the magnitude distribution index may have been slightly steeper. From Kai Gaarder's magnitude estimates, we have $r=2.8$ (Jenniskens 1988).

\section{ANTICIPATED ACTIVITY IN DECEMBER 2000}

We submit that the broad 1981-1982 and 1993-1997 perihelion outbursts are probably the accumulation of large dust grains from multiple debris trails, trapped in the same mean motion resonance as that of the parent comet, forming a structure much like the Perseid and Leonid "Filaments" (Jenniskens et al. 1998, Jenniskens and Betlem 2000). This accumulation has occurred over a relatively long period of time. For example, Brown and Arlt (2000) derived an accumulation age stretching from AD 400 until 1700 for the Leonid Filament. In our opinion, this results from particles catching up on each other by small perturbations of the orbital period. In the process, the signature of individual dust trails is lost and nearby particles can have significantly different orbital elements.

\section{The 8P/Tuttle Dust Trails}

Given the much more narrow width of the aphelion outbursts, on the other hand, it is not unreasonable to argue that the material responsible for the 1986 outburst is not very old and can probably be traced back to a single dust trailet. Similarly, the 1945 outburst might well be identified with a single dust trailet.

That hypothesis has the exciting prospect of predicting possible Ursid outbursts in December of 2000 and beyond, analogous to recent predictions of Leonid returns (Lyytinen and

TABLE II

Ursid Orbits (J2000) from the 1945 Outburst (Ceplecha 1951—Recalculated) and the 1997 Ursid Outburst

\begin{tabular}{|c|c|c|c|c|c|}
\hline Year & $\begin{array}{c}1945 \\
3 \text { single station } \\
\text { (aphelion outburst) }\end{array}$ & $\begin{array}{c}\text { 8P/Tuttle } \\
1994\end{array}$ & $\begin{array}{l}1997 \text { (Filament) } \\
\text { median of } 10 \text { orbits } \\
\text { (perihelion outburst) }\end{array}$ & $\begin{array}{c}2000 \\
59 \text { orbits } \\
\text { (aphelion outburst) }\end{array}$ & $\begin{array}{l}\quad 2000 \\
1405 \\
\text { trail model }\end{array}$ \\
\hline Date & Dec. $22.773 \pm 0.051$ & June 17.0 & Dec. $22.434 \pm 0.057$ & Dec. $22.32 \pm 0.06$ & Dec. $26 \pm 3$ \\
\hline$R A$ geo & $217.06 \pm 0.07$ &.-- & $222.1 \pm 4.2$ & $219.0 \pm 4.6$ &.-- \\
\hline$D E C$ geo & $75.63 \pm 0.05$ &.-- & $75.0 \pm 0.5$ & $75.3 \pm 1.8$ &.-- \\
\hline$V$ geo & 33.47 (assumed) &.-- & $32.25 \pm 0.87$ & $33.05 \pm 1.1$ &.-- \\
\hline$a$ & 5.716 (assumed) & 5.671775 & $4.62 \pm 0.93$ & $4.673 \pm 0.98$ & $5.793 \pm 0.002$ \\
\hline$e$ & $0.8363 \pm 0.0015$ & 0.824088 & $0.795 \pm 0.040$ & $0.799 \pm 0.053$ & $0.8378 \pm 0.0001$ \\
\hline$q$ & $0.9357 \pm 0.0002$ & 0.997732 & $0.944 \pm 0.006$ & $0.940 \pm 0.009$ & $0.9398 \pm 0.0006$ \\
\hline incl & $53.10 \pm 0.03$ & 54.69254 & $51.5 \pm 1.1$ & $52.5 \pm 1.9$ & $52.96 \pm 0.02$ \\
\hline$\omega$ & $206.73 \pm 0.04$ & 206.7028 & $204.9 \pm 2.0$ & $205.9 \pm 2.8$ & $205.68 \pm 0.11$ \\
\hline$\Omega$ & $271.35 \pm 0.05$ & 270.5487 & $270.64 \pm 0.06$ & $270.76 \pm 0.06$ & $270.7579 \pm 0.0044$ \\
\hline
\end{tabular}


Van Flandern 2000). In recent years, relatively simple meteoroid stream models have been developed that have proven very successful in predicting the encounter of Earth with comet dust trails 1-4 revolutions old but have given poorer results for dust trails 59 revolutions old (Kondrat'eva and Reznikov 1985, McNaught and Asher 1999, Lyytinen 1999).

The model integrates a small range of single particle orbits with sufficient difference in orbital period (or semi-major axis a) to achieve a lag that matches the time difference between the return of the comet to Earth's orbit and the observation of the meteors at the time of encounter. In our model, that lag is caused by radial radiation pressure effects. Others have included an initial ejection at perihelion in forward direction, resulting in the nearly the same initial orbit (Kondrat'eva and Reznikov 1985, McNaught and Asher 1999).

We were now ready to take that model a step further and consider dust trails of several tens of orbits old. Comet 8P/Tuttle has an orbit outside of Earth's orbit. For a meteoroid to hit Earth, the particle's perihelion distance $(q)$ has to move inward to the Sun. Our investigation of the orbital dynamics of the Ursid meteoroids shows that only trails no younger than several hundred years back intersect Earth's orbit today.

Recently, we worked to improve the agreement for the somewhat older Tempel-Tuttle dust trails by including nongravitational effects (Lyytinen and Van Flandern 2000). We found that the Poynting-Robertson effect and the diurnal Yarkovsky effects are small on the time scales involved in our problem. Rather, we suspect that the seasonal Yarkovsky effect may continue to accelerate the grains over time. Such continuous acceleration (the "A2 effect" in Lyytinen and Van Flandern 2000) is simulated by slightly changing the speed of the meteoroids at each perihelion, not the direction of motion. In the Comet 8P/Tempel dust trail model, we did not cover the full parameter space of possible values, but rather examined cases without and with such correction, for the amount found to best match our Leonid observations in 2000 (Lyytinen et al. 2001).

As before, we calculated the orbital evolution of dust trails ejected over the past 800 years for a range of semimajor axis covering the orbital resonances close to that of the comet. Initial orbital elements were those of the parent comet at perihelion with only the semi-major axis adjusted. All planets were included in the calculation, except Pluto. The J2000 planetary orbits were adopted from the Jet Propulsion Laboratory (JPL) database. The comet orbit was taken from the International Astronomical Union (IAU) Minor Planet Center database. The comet and planet orbits were backward integrated for 2000 years, allowing for the continuous acceleration term and for the gravitational effects of the planets. We used an orbit integrator of our own design. The results were checked by Hartwig Leuten (Personal communication, 2000) using a model including both the normal radial pressure and a continuous acceleration term and using the K11 orbit integrator, version 3.0 by Christian Clowinski. We find that the three independent numerical integrations are consistent with one another, with less than $0.0001 \mathrm{AU}$ difference between the radial distance of dust trail and Earth $(r \mathrm{D}-r \mathrm{E})$ after 46 revolutions.

We plotted the resulting differences between the radial distance of dust trail and Earth $(r \mathrm{D}-r \mathrm{E})$ as a function of the ecliptic crossing time; this type of plot gives a rapid overview of even the most chaotic trails. For example, Fig. 4 shows the position of the dust particles of the 1392 dust ejecta in time of ecliptic plane crossing (horizontal axis) and space in terms of $r \mathrm{D}-r \mathrm{E}$ around the time of the December 1945 outburst. In contrast to the recent Leonid ejecta, these ancient dust trails are far from well-behaved lines. Close encounters with Earth cause frequent gaps. The mean-motion resonances sometimes help preserve trail fragments (trailets), but in other cases a chaotic cloud of particles is all that remains.

Inspecting the historic variations of $q$ of the parent comet, we find maybe a dozen returns that could have resulted in a dust trail close to Earth's orbit at the present time. We examined a number of those trails to identify the ones likely to cause an outburst. Of course, many trails from encounters further back in time may contribute as well, but those should be less significant.

The 1945 outburst appears to have been caused by the dust ejected in 1392, for which the miss distance was less than $r D-$ $r E=+0.001 \mathrm{AU}$ (Fig. 4). The dust trail is still well behaved at the point of intersection, indicated by an arrow. The computed encounter time (the node of the particle pointed to by the arrow), Dec. 22, 18:29 UT, is compatible with the meteor stream activity curve plotted in Jenniskens (1995), although the descending arm of this curve remained unobserved. Integration forward to 2000 shows that this same trail can perhaps produce a meteor outburst in December 2000 at solar longitude 270.808 (J2000). However, the miss distance $(r \mathrm{D}-r \mathrm{E})$ has increased to $+0.0038 \mathrm{AU}$.

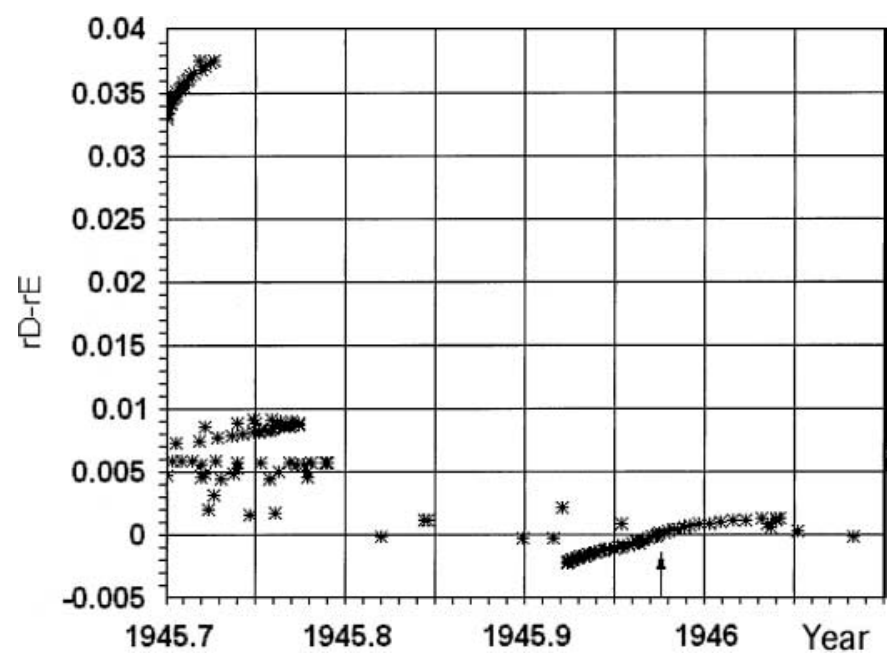

FIG. 4. The 1392 dust trail in a diagram showing along the horizontal axis the point in time when the particles cross the ecliptic plane (in years) and along the vertical axis the distance from Earth's orbit (in astronomical units), around the time of the December 22, 1945 Ursid outburst (arrow). The Sun is in a downward direction. 


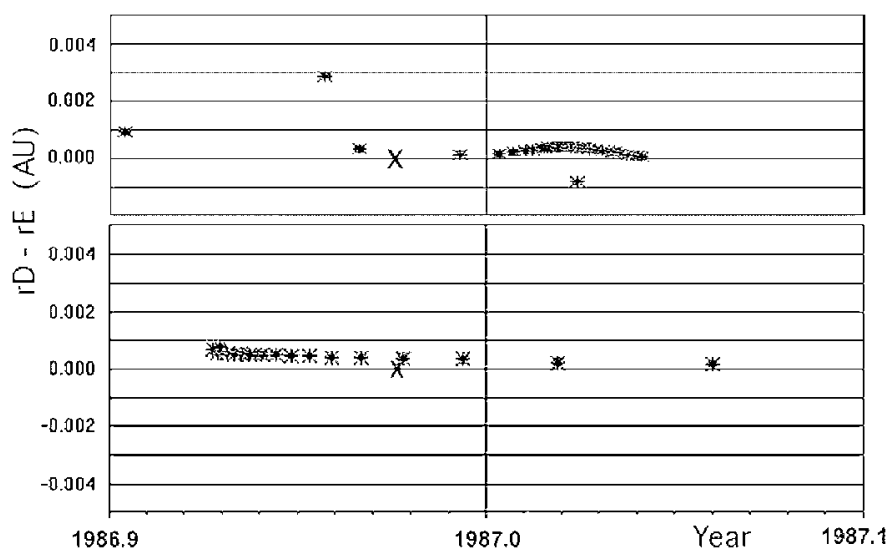

FIG. 5. As in Fig. 4, for two trail fragments of the dust ejected in 1378. The "X" marks the Earth encounter during the December 22, 1986 (1986.975) Ursid outburst. The upper part is more densely sampled than the lower part.

The 1986 outburst appears to have been caused by a trail from dust ejected 44 revolutions back in the year 1378 (Fig. 5). The trail comes close to Earth's orbit and the timing is to within $0.01^{\circ}$ from the observed value. This particular trail folds on itself, and a higher local density of model particles than normally used is needed to get a good view of the position of the trail near Earth's orbit. Figure 5 shows two small trail fragments that approach Earth's orbit at the time of the December 1986 Ursid outburst. The first trailet (top Fig. 5) shows a regular course, with the exception of two test particles. The orbital elements of the particles near the intersection ("X") have a node at 270.934 (J2000), which is close to the peak of Fig. 3. The trailet is stretched out significantly at the point of intersection, which would have lowered the expected dust density. We conclude that if the encounter had been a few weeks later, the Ursid shower would have been more intense. The second fragment of the 1378 trail (lower part of Fig. 5), particles with a higher original $\Delta a$, does also encounter Earth at the expected time. The encounter is at 270.816 (2000.0). The trail is less stretched out, but the dust density is expected to fall off exponentially with original $\Delta a$, making this trailet a factor of 10 less important (Lyytinen and Van Flandern 2000). The observations are not precise enough to detect this feature. Both segments of the 1378 trail are near Earth's orbit as well in December of 2000, when the encounter occurs at solar longitude 270.82 (J2000), but at a distance of $+0.0069 \mathrm{AU}$ and at $+0.025 \mathrm{AU}$ for the two trailets, respectively.

The more likely candidate for an outburst in 2000 is the next 45 revolution old trail of 1405 . The denser model in Fig. 6 shows that a well-behaved trail segment is near Earth's orbit at that time. The particle at the tip has a small $r D-r E=-0.00124 \mathrm{AU}$ at solar longitude 270.7542 (J2000). The trail curves on itself right at that point in time and the local dust density is relatively high. In our initial study (Jenniskens and Lyytinen 2000), we did not include continuous acceleration in the model and noticed that this part of the dust trail that curves in on itself did not reach the point in time of the encounter. If true, the dust would only have started crossing the Earth's orbit 1.4 days too late. However, after including the continuous acceleration effect, we can confirm that the dense tip of the 1405 dust trail has moved to cover the outburst date, while decreasing $r D-r E$ (line in Fig. 6, marked "a"). In fact, a continuous acceleration of only $3 \%$ of that needed to best fit the 2000 Leonid outbursts is sufficient to bring the dense tip in the Earth's path.

Figure 7 summarizes the calculated position of the various dust trails in December of 2000. The view is from the north ecliptic pole, with the Earth moving right to left and the Sun in a downward direction. Each ellipse is the expected two-timesFWHM trail density contour in projection on the ecliptic plane, after taking into account the inclination of the orbit and the relative dimensions measured for the 55P/Tempel-Tuttle dust trails. Those trails are found to be a factor of 3.0 wider in the plane of the comet orbit than in the path of the Earth (Jenniskens 2001b, updated from "factor of 4" in Jenniskens and Gustafson 2000).

\section{The Cause of the 6-year Lag}

For a given dust trail, the majority of particles are so confined in mean anomaly that they reach Earth only in the year of the anticipated outburst, with no particles near Earth's orbit in the year before or after. This is shown in Fig. 8, where each symbol is a particle from one of the dust trails ejected in 1365, 1378, 1392 , or 1405, and each point represents the crossing of a particle through the ecliptic plane at the given time ( $x$ axis) and at the given miss distance $r D-r E$. The $y$ axis $r D-r E=0$ corresponds to Earth's position on December 22 in the given year.

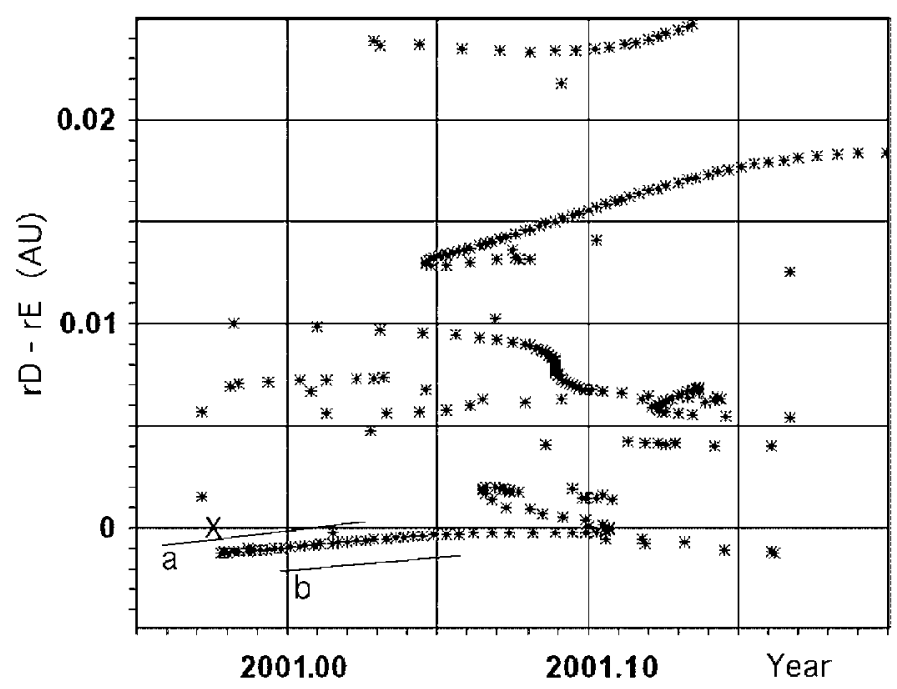

FIG. 6. As in Fig. 4, for the 1405 dust trail. The "X" marks the encounter during the December 22, 2000 (2000.975) return. Solid lines marked "a" and "b" show the trail position for continuous accelerations of -1.0 (a) and +1.0 (b) millionths of the speed at perihelion. 


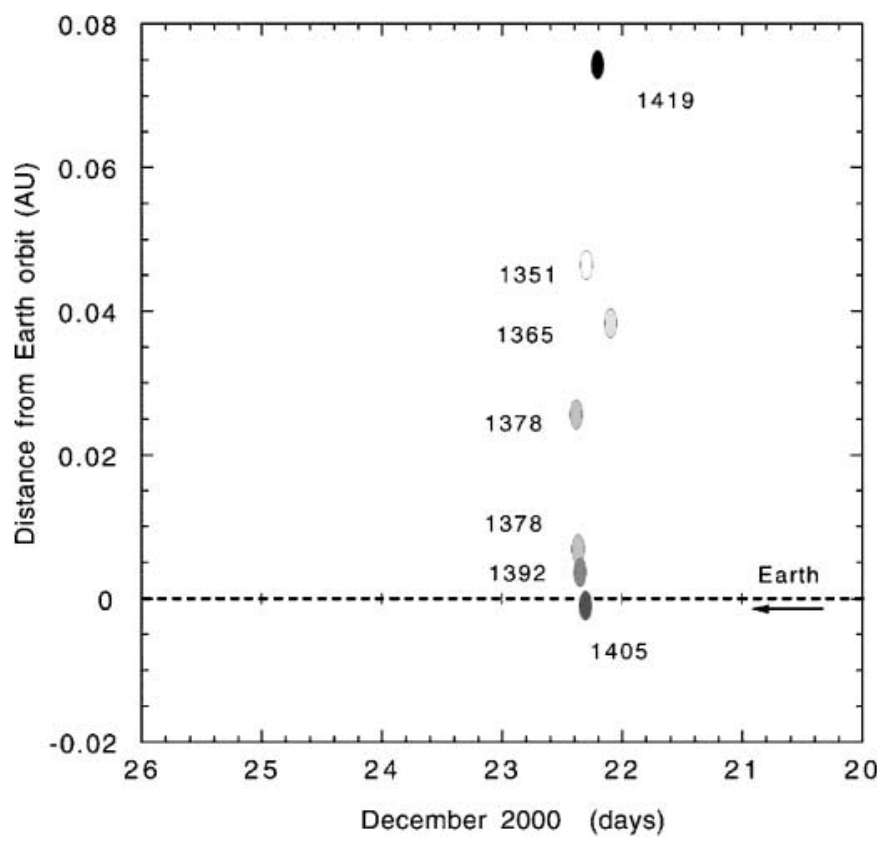

FIG. 7. Position of the various dust trails of 8P/Tuttle relative to Earth's orbit (dashed line) in December 2000. The size of the ellipses in twice the expected full-width-at-half-maximum. Older trails are marked in lighter shades.

The model also explains why particles encounter Earth only 6 years after the passage of the comet. The comet's passage by the node is marked in Fig. 8. The reason for this is the mean motion resonances. The comet currently librates around the "high" 13:15 mean motion resonance with Jupiter. Because of radiation pressure effects on the particles, they tend to get into slightly longer orbital periods than the comet and end up trapped in the mean motion resonance 12:14 (=6:7). Because it takes the particles longer to travel around the Sun, they will gradually move away from the comet. If the resonant librations have small enough amplitude to keep the particles near the cen- ter of the "window," then there will be a systematic decrease of the perihelion distance $(q)$ that is faster than for the comet itself. This will ultimately lead to meteoroid Earth crossings as needed, with the comet orbit just outside Earth's orbit. Indications are that this decrease of $q$ is the result of planetary perturbations from Saturn, and to a lesser extent from Uranus and Neptune. Note, however, that near the leading edge of the resonance, the particles may have close encounters with Jupiter that will increase $q$ instead.

We find that it takes about six centuries for dust to move close to Earth's orbit. During that time, the separation of the particles and comet in mean anomaly, as a result of the difference in orbital period, increases to 6 years. This is a natural explanation of why the 1986 and 1945 outbursts occurred about 6 years after the comet's closest encounter with Earth's orbit, or near aphelion. We also find that the resonances effectively confine the dust to a single year return, thus explaining the lone nature of the outbursts.

Younger trails than that from 1405 are found nowhere near Earth's orbit in December of 2000 (Fig. 7). The 1419 trail has $r \mathrm{D}-r \mathrm{E}=+0.075$, while the next trail from 1433 has $r \mathrm{D}-$ $r \mathrm{E}=+0.084 \mathrm{AU}$. The big difference between the trails from 1405 and 1419 (Fig. 7) is caused by a Jupiter encounter at about $0.86 \mathrm{AU}$ of the parent comet in 1462 . Between the 1460 and 1474 returns, the perihelion distance of the comet increased +0.026 AU. The particles released in 1419 (that cross the ecliptic at the descending node near December 22, 2000) are about 2 months behind the comet in 1462 and in part share this increase, more so than particles released in 1405. As the difference in $r \mathrm{D}-r \mathrm{E}$ between both trails is now bigger than about $0.02 \mathrm{AU}$, it may suggest that the Jupiter encounter sets the particles from 1419 into an unfavorable librational status. The dust particles that escaped before this into a longer period orbit made a more distant encounter and so were put in favorable conditions to decrease the perihelion distance. The earlier the particles were ejected, the bigger this advantage. Particles ejected several revolutions earlier,

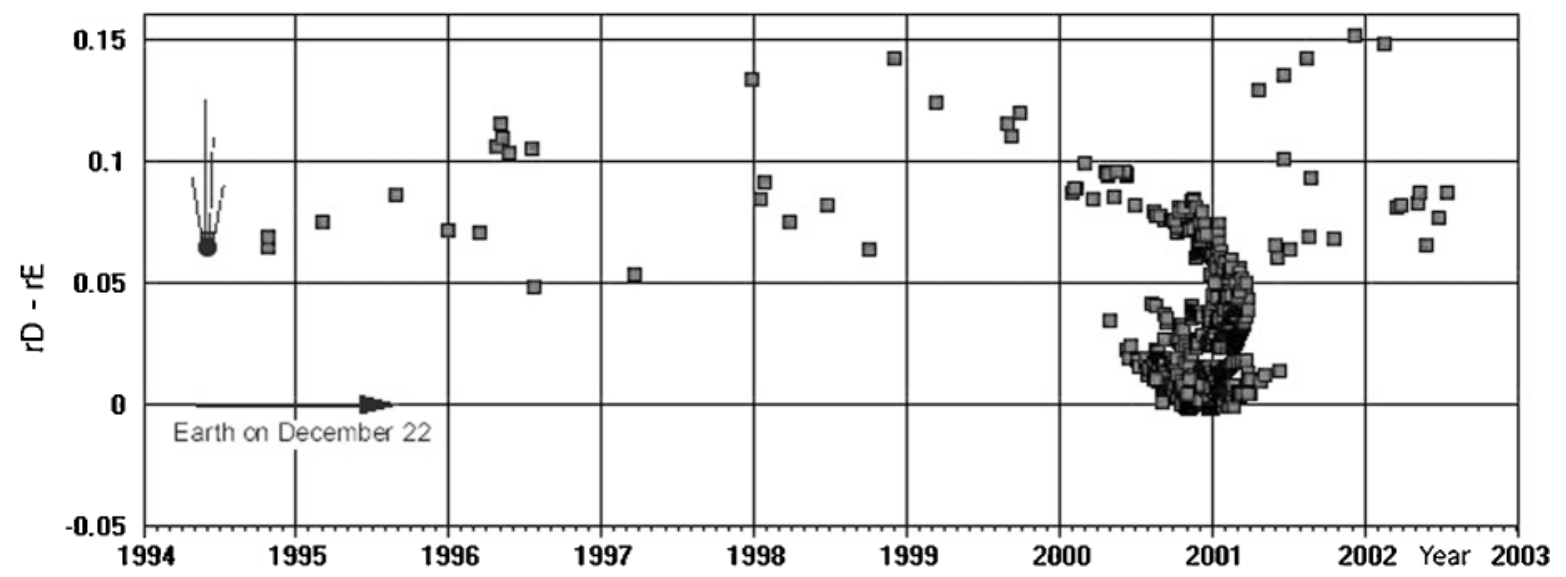

FIG. 8. Data as in Figs. 4-6, in a compilation of test particles for the trails of 1365, 1378, 1392, and 1405. The figures shows that resonances effectively confine the dust to a narrow stream seen only in December 2000 (2000.975), 6 years after the comet's ecliptic plane crossing (marked by a comet symbol). 
however, got into other unfavorable circumstances. Hence, there was this region of a few revolutions that seemed to be both the most favorable to get the particles into the librational window in question and a good start to reach as small a perihelion distance as needed.

\section{Specific Predictions for the 2000 Encounter}

Based on these calculations, we made the following specific predictions for the December 2000 encounter (Jenniskens and Lyytinen 2000):

(1) The most likely trail passage would be with the trail of 1405, expected to peak at 7:29 UT. Figure 7 shows that Earth crosses the 1405 trail within the two-times-FWHM of peak intensity contour. Because the particles of the 1405 trail have relatively high initial $\Delta a$, they are expected to be smaller than during past outbursts in 1945 and 1986, perhaps rather near the visual detection limit under good observing conditions. The smaller particles have higher surface-to-mass ratio and thus higher ejection velocities and higher radiation pressure effects.

(2) If the dust trail width in the plane of the comet orbit is wider, perhaps due to a range of continuous acceleration acting on the particles, or the trail positions are slightly shifted with respect to the calculated positions, then the trailet of 1392 might show up at 8:38 UT. If so, these events probably would make a continuous profile $4-5 \mathrm{~h}$ wide, but could perhaps be recognized separately. Both events favor northern hemisphere observers in the Americas. The Moon would be out of the way, providing for generally good observing conditions.

(3) The expected intensity of the shower was hard to predict, not in the least because of the necessary influence of a continuous acceleration effect. Both the historic 1945 and 1986 encounters had miss distances $r \mathrm{D}-r \mathrm{E}<0.001 \mathrm{AU}$, but the dust densities in the trail according to the mean anomaly factor (which quantifies the extent to which trail particles have separated in mean anomaly, as reflected in the spreading of the particles in Figs. 4-6) seemed to be smaller than in this year. The density in the 1405 trail, however, was relatively large. Because of that, we expected peak rates similar to past outbursts, on the order of 1 per min.

(4) Although calculated to be far from Earth's orbit, the 1378 dust (at 8:59 UT, Fig. 7) cannot be ignored, because this trail shows a relatively rapid increase of $r D-r E$ toward the end of 2000 (figure not shown). Since there are particles around $r D-r E=0$ only about a month or two earlier, it may be that the continuous acceleration effect could bring those in Earth's path.

\section{OBSERVATIONS OF THE 2000 ENCOUNTER}

In order to confirm the predictions, observing teams were assembled in California to deploy intensified-video cameras and photographic cameras at two separate sites for multistation imaging. Low-resolution spectrographs were deployed as well, in the hope of comparing the physical properties of the Ursid meteoroids with those of the Leonids.
In search of clear weather, the two sites were set up at a 1-h further drive south than our usual observing sites south of the Bay Area. At Lake San Antonio just south of King City, Mike Koop operated four intensified video cameras (two aimed at $70^{\circ}$ elevation and two at $30^{\circ}$, due east). Mike Wilson ran a CCD spectrometer, and Chris Angelos and Peter Gural operated a 13-camera 35-mm photographic setup and an all-sky intensified video camera for meteor timing. At a site $74 \mathrm{~km}$ to the east, south of Coalinga at the intersection of routes 33 and 41, Peter Jenniskens operated six intensified cameras (four at $70^{\circ}$, two at $25^{\circ}$ east) and the low-resolution slitless spectrograph "BETSY," while Ming $\mathrm{Li}$ and Duncan McNeill ran a second 13-camera battery.

Corroborating observations were obtained in the Netherlands, where Casper ter Kuile, Klaas Jobse, Robert Haas, and Romke Schievink of the Dutch Meteor Society operated intensified video cameras. The first three observers set up cameras from their home locations in De Bilt (52 07' N, $\left.511^{\prime} \mathrm{E}\right)$, Oostkapelle (51 $\left.34^{\prime} \mathrm{N}, 332^{\prime} \mathrm{E}\right)$, and Alphen a/d Rijn (52 09' N, $\left.440^{\prime} \mathrm{E}\right)$, while Romke traveled to the Public Observatory Twente in Lattrop $\left(5225^{\prime} \mathrm{N}, 658^{\prime} \mathrm{E}\right)$. The radiant altitude was around $60^{\circ}$. All cameras applied second-generation image intensifiers and $\mathrm{Hi} 8$ or digital camcorders. During the observations, the cameras were pointed at the same spot in the atmosphere to allow for multistation detections. The images from the camera in Alphen a/d Rijn were not useful for stereoscopic measurements because of a misalignment, but were included in flux measurements.

\section{Ursid Activity}

In The Netherlands, visual observers noticed a gradual increase in Ursid rates, marking the onset of the outburst. Fifteen Ursid orbits were calculated (DeLignie and Johannink 2001) and are listed in Table I, with a code name starting with "205." When twilight started interfering, California observers took over. Rates gradually increased, until a peak at about 8 UT. A total of 431 Ursids were detected in a single visual scan of the videotapes amidst 394 other meteors in a total of $28.4 \mathrm{~h}$ of effective observing time. Orbital elements were calculated for the best 42 Ursids (between 6:45 and 9:08 UT), the brightest only magnitude +1 . Results are listed in Table I under code names starting with "207." Despite a significant effort, only two Ursids were photographed and both were not multistation and only just bright enough to be detected. Four Ursid spectra were recorded with the slitless spectrograph, all faint. No Ursid was bright enough and well enough placed to give a good signal on the CCD spectrometer.

Figure 9 shows the average number of Ursids counted in 10-min intervals from eight independent cameras, six at the Coalinga site and two at the King City cite $(\bullet)$. These counts are scaled to the zenith hourly rates calculated from visual observations in The Netherlands ( $\circ$, left), and similar observations in Japan, calculated by Masaaki Takanashi of the Nippon Meteor Society (NMS electronic circular, Dec. 2000). Radio meteorscatter observations were provided by five stations of the Global Meteor Scatter Network (Jenniskens 1998b). Ten-minute counts 


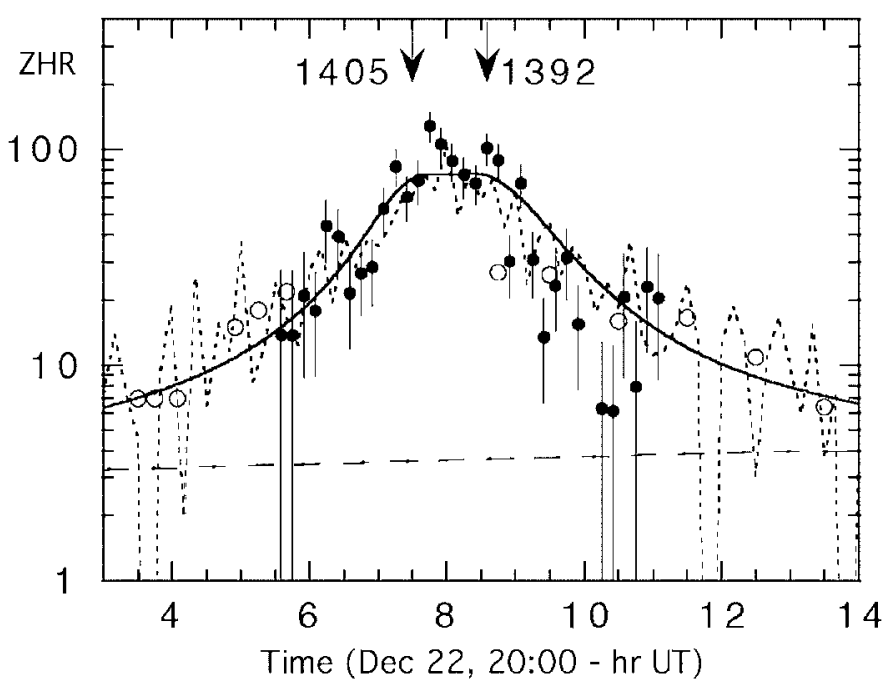

FIG. 9. Activity curve of Ursids from video $(\bigcirc)$, visual $(\bigcirc)$, and radio forward meteor scatter data (- - ) in units of zenith hourly rate (ZHR). The broadly dashed line near the bottom shows the level of annual Ursid activity. The solid line is the Lorentzian profile fitted to the data. The expected peak times for the 1405 and 1392 dust trails are indicated.

were obtained by Esko Lyytinen and Ilkka Yrjölä in Finland, Hiroshi Ogawa and Kazuhiro Suzuki in Japan, and Pierre de Groote in Belgium. The mean of those counts is shown as a dashed line in Fig. 9 and corresponds well with the video and visual record.

A Lorentzian curve fitted to the data gives a full-width-athalf-maximum of $W=0.09 \pm 0.01^{\circ}$ (or a duration of 4-5 h) and a peak time at solar longitude $=270.780 \pm 0.005$ (J2000), or $8 \mathrm{~h} 06 \pm 07 \mathrm{~m} \mathrm{UT}$. The peak activity appears to have been rather high, with $Z H R \sim 90$, but the high magnitude distribution index and the low radiant altitude $\left(26^{\circ}\right)$ made this a much less impressive shower than the Perseids. From the ratio of sporadics and Ursids, we find $r=3.5 \pm 0.5$ before $8 \mathrm{~h} \mathrm{UT}$ and $r=2.8 \pm$ 0.3 after $8 \mathrm{~h}$ UT, assuming all other meteors (sporadics) have $r_{\mathrm{s}}=3.4$ (Fig. 10). For the high cameras only, we have $r \sim 3.2$ and $r \sim 2.9$, respectively. From the Ursid count as a function of magnitude, we have $r \sim 2.6$ and $r \sim 2.4$, with $r_{\mathrm{s}} \sim 3.0$, but this result is sensitive to the assumed magnitude range over which all meteors are detected.

\section{Ursid Trajectories and Orbits}

Until our work, only one Ursid orbit had been calculated from multistation observations and included in the IAU Meteor Data Center database (Lindblad and Steel 1994). Now, there are sufficient data to study the meteoroid dispersion. The distribution of radiant positions (defining the orientation of the entry velocity vector of individual meteoroids) is significantly dispersed in right ascension and (less so) in declination (Fig. 11). There are some possible groupings, with a cluster of radiants at relatively low $R A<215^{\circ}$ and another grouping at $D E C<75.0^{\circ}$. The median radiant position and orbital elements are listed in Table II.

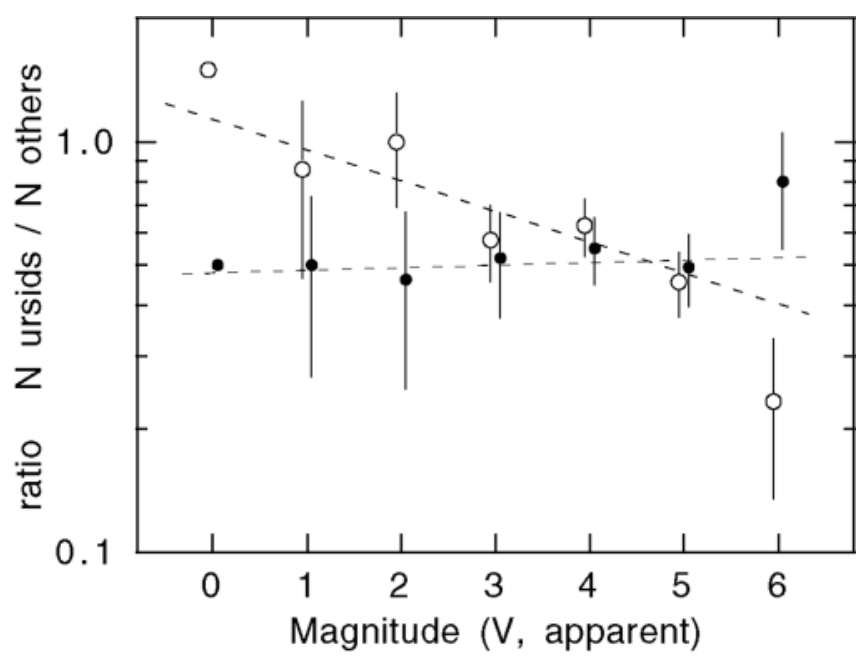

FIG. 10. Apparent magnitude distribution as manifested in the ratio of Ursids and Sporadics for periods prior to $(\bullet)$ and after $(\bigcirc)$ 8H UT (all cameras). Shown is the ratio of the count of Ursid versus that of sporadic meteors in each magnitude interval.

The speed of the meteors scatters around $32.7 \mathrm{~km} / \mathrm{s}$ (Fig. 12), but radiants with low right ascension have systematically higher speed $(34.2 \mathrm{~km} / \mathrm{s})$. Also, radiants from 1997 that have unusually high right ascension tend to have lower speed $(31.9 \mathrm{~km} / \mathrm{s})$.

As expected, there is a strong concentration of orbits near $1 / a=0.1734$ (6:7), shown in Fig. 13. The distribution may perhaps peak at a slightly higher $1 / a=0.21$, but that could mean simply a systematic error in the velocity measurements of $-0.29 \mathrm{~km} / \mathrm{s}$ in $V_{\text {inf. Possible concentrations at } 1 / a=0.28}$ and $1 / a=0.15$ represent velocity differences of $\sim-0.8$ and $+0.2 \mathrm{~km} / \mathrm{s}$, respectively. The speed of the meteors may contain a systematic error because there is an unknown difference between the mean velocity $\langle V\rangle$ and the entry velocity before atmospheric deceleration $V_{\text {inf }}$. We have adopted a constant difference

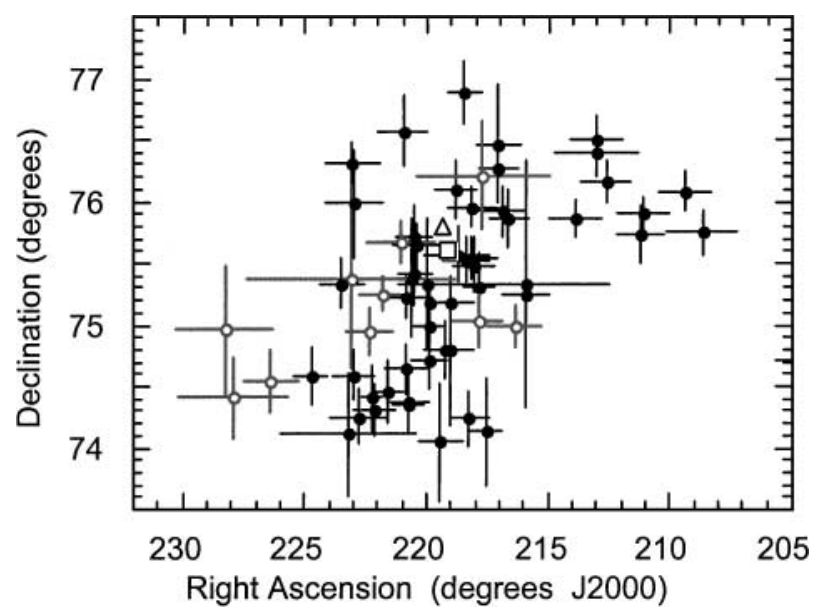

FIG. 11. Ursid radiant positions measured during 2000 outburst (filled circles) compared to those measured during 1997 outburst (open circles). An open square and triangle show the expected radiant positions for the 1405 and 1392 dust trails of 8P/Tuttle, respectively. 


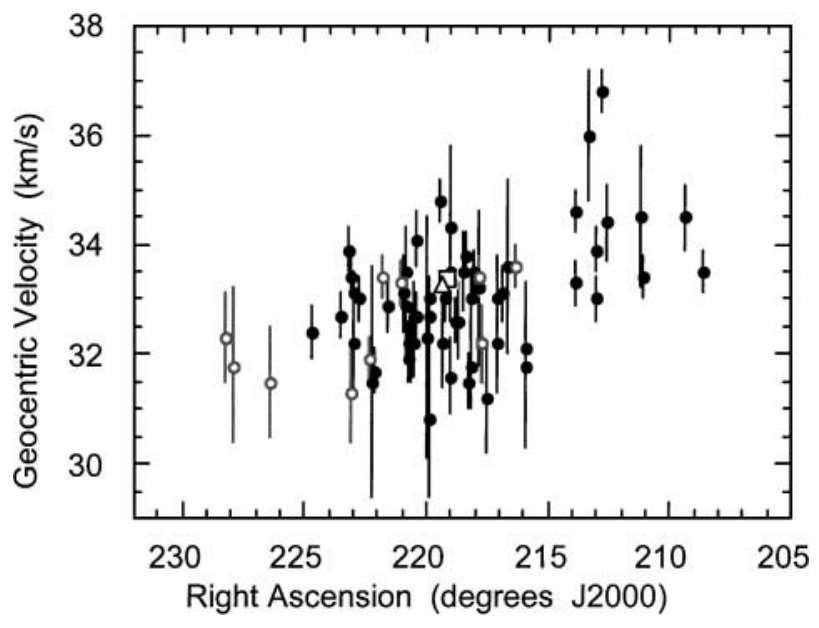

FIG. 12. As in Fig. 11, for the distribution of measured speed as a function of the direction of the velocity vector (radiant) in terms of right ascension.

of $V_{\mathrm{inf}}-\langle V\rangle=+0.34 \mathrm{~km} / \mathrm{s}$, consistent with other meteors of similar entry velocity.

While the perihelion distance $(q)$ and argument of perihelion $(\omega)$ are mainly determined by the measured radiant position, the inclination $(i)$, eccentricity $(e)$, and semimajor axis $(a)$ are mostly determined by the measured speed. Figure 14 plots the inclination versus perihelion distance for the most precise orbits $\left(\sigma i<0.55^{\circ}\right)$. Only representative error bars are shown. There is a separate cluster of orbits with significantly lower perihelion distance. Those correspond to the low $R A<215^{\circ}$ (Fig. 11) and high velocity (Fig. 12) outliers.

\section{Physical Properties of Ursid Meteors}

The outburst Ursids show various signs of a more fragile than usual morphology. This is a familiar property of recent

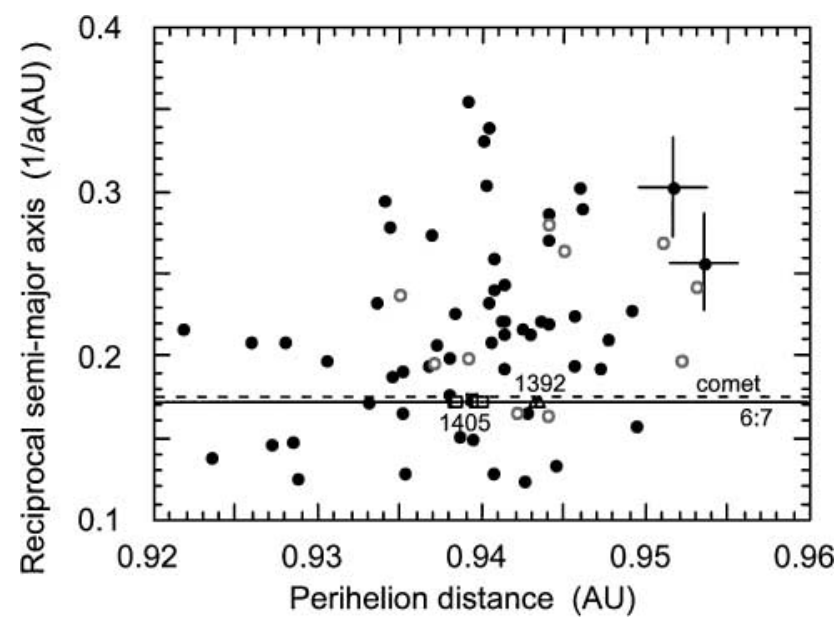

FIG. 13. Symbols as in Fig. 11, for the distribution of semimajor axis versus perihelion distance. Crosses show a typical measurement error. Dashed lines indicate the location of the mean motion resonances.

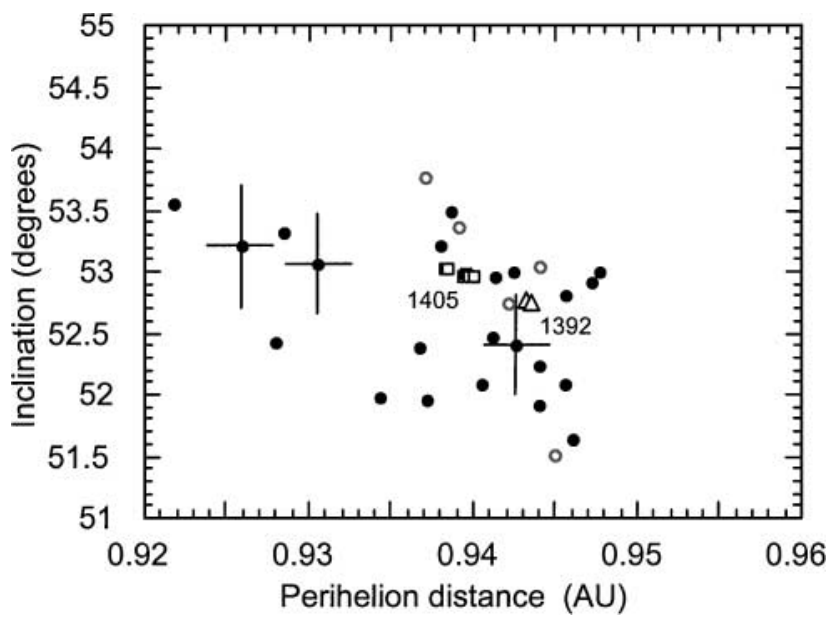

FIG. 14. Symbols as in Fig. 11, for the distribution of orbital elements in a subset of orbits with precision better than $\sigma i=0.55$ degrees. Crosses show typical measurement errors.

Giacobinid ejecta (Draconids) and Tempel-Tuttle ejecta (Leonids). Apparently, this morphology is not much affected even after 44 revolutions or a total age of about 600 years in the interplanetary medium for an orbit with $q \sim 0.94$. Specifically, we find that the Ursid meteoroids do not penetrate as deep as observed for sporadic meteors and other showers at similar entry velocity. Beginning and end heights are summarized in Fig. 15. The best Ursid spectrum at 08:24:54 UT (Fig. 16) shows an early release of sodium relative to magnesium in a similar manner as observed before for the storming Leonids and Draconids (Borovicka et al. 1999). It is thought that the sodium-containing minerals are exposed more effectively to the impinging air molecules, or the sputtering plasma, than in normal meteors. One explanation is that the meteoroids fall apart in small fragments that expose the volatile minerals more efficiently.

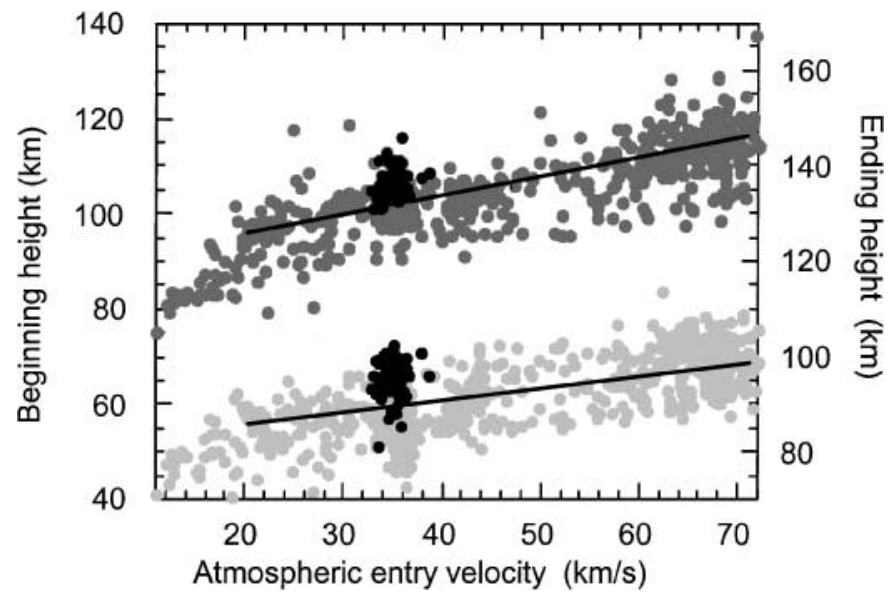

FIG. 15. The beginning and end height of Ursid meteor trajectories (black) in relation to other meteors in our database (as of 1998) that were recorded with the same intensified video cameras. 


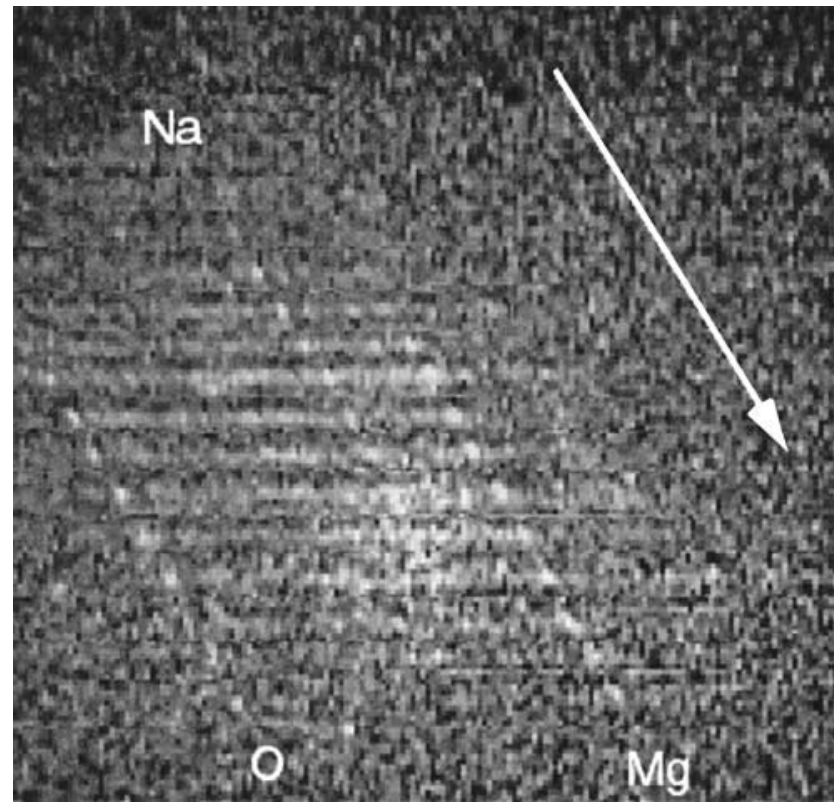

FIG. 16. Compilation of the video spectrum of the 08:24:4 UT Ursid meteor. The wavelength scale runs right to left, while the meteor descended in altitude from top to bottom in the direction of the arrow (outside field of view). Individual frames show the emission lines of atmospheric oxygen $(\mathrm{O})$, meteoric sodium $(\mathrm{Na})$, and meteoric magnesium $(\mathrm{Mg})$ atoms. Diffuse horizontal bands are the first-positive bands of atmospheric molecular nitrogen.

Another interesting result is the relative strength of the atomic emissions of nitrogen and oxygen and the first positive bands of molecular nitrogen, which is a very sensitive thermometer for air plasma equilibrium temperatures $T$ (Jenniskens et al. 2000b):

$$
\frac{I(\mathrm{OI}, 777 \mathrm{~nm})}{I\left(\mathrm{~N}_{2}, 700-776 \mathrm{~nm}\right)}=8.85 \times 10^{-14} \exp \left(0.00676^{*} T\right) .
$$

The presence of the $\mathrm{N}_{2}$ bands (the diffuse emission between the $\mathrm{Mg}, \mathrm{Na}$, and $\mathrm{O}$ lines) implies that the chemical equilibrium temperature for the $\mathrm{N}+\mathrm{N} \leftrightarrow \mathrm{N}_{2}$ reaction in the meteor wake is not much different from that observed in Leonid meteors that are double the speed of Ursids. While Leonid meteors of about magnitude -1 have $T=4300 \pm 100 \mathrm{~K}$, the spectrum of this +2 Ursid results in $T=4260 \pm 200 \mathrm{~K}$.

\section{DISCUSSION}

For the first time, the link between the Ursid shower and 8P/Tuttle has been established beyond doubt. Until now, the association of the Ursids with Comet 8P/Tuttle depended on the comparison between meteoroids hitting Earth and a comet that passed far from Earth's orbit. That situation does not change with the new measurements. However, now we can compare the Ursid orbits to the median orbit of the test particles in the 1405-dust trail model. In Figs. 11-14, the orbital elements of test particles representing the two dust trails of 1405 and 1392 are indicated with open symbols. The observed orbits scatter around the calculated positions of both trails. We find good agreement in all orbital elements, within the error bars.

The return of the Ursids demonstrates that the basic approach of current models is valid even for trails dating back as many as 45 orbital revolutions. Specific showers can be identified with dust from specific returns of the comet. For the first time, we have proof that mean motion resonances are important in the evolution of cometary dust trails from Halley-type comets. The 6-year lag can only be understood if a systematic difference between orbital periods of comet and meteoroids is maintained and the meteoroid orbital periods are confined in a small range.

Concerning the details of the encounter, we note that the 1378 trail was not detected. The observed activity profile peaks exactly in between the predicted times for the 1405 and 1392 dust trails, as expected if both trails contribute to the profile, and the radiant position scatters around the expected positions of both trails. Indeed, the magnitude distributions may suggest a slightly brighter 1392 component after the peak. If both trails were present and the peak times were correct, then they contributed equal amounts within $\sim 30 \%$ (solid line, Fig. 9). The activity curve is not double-peaked, which puts the width of each trail (assumed to be equal) at $W=0.06 \pm 0.01^{\circ}$, in good agreement with the $W=0.05 \pm 0.01^{\circ}$ for the 1378 dust trail (1986 outburst). For both trails to contribute to the shower, the dispersion perpendicular to Earth's path (in the plane of the comet orbit) must be significantly larger than that in the Earth's path, by at leas a factor of 3 , as for the younger Leonid dust trails (Jenniskens 2000b).

The equal contribution implies that the 1405 and 1392 trail centers were slightly more inward to the Sun than calculated, by about $-0.0013 \mathrm{AU}$. This is a similar, but smaller, shift than was observed for the 1-9 revolution old Leonid dust trails $(-0.00025 \mathrm{AU})$ and attributed to a nonnegligible heliocentric ejection velocity component (Jenniskens 2001b). Alternatively, the 1405 trail may have been slightly less shifted backward in time from the assumed continuous acceleration effect while the 1392 dust trail could be more spread out perpendicular to the Earth's orbit than the factor of 3 derived for the younger Leonid trails.

To our surprise, we do not find a more compact radiant structure for the 2000 outburst Ursids, compared to that for the "Filament" component observed in 1997. Only 23 orbits (50\%) cluster around the theoretical geocentric radiant position and speed of the 1392 and 1415 trails. Eight orbits (17\%) are clearly outliers with systematically lower $R A$ and higher $V_{\text {inf }}$. Although these orbits were mostly observed after $8 \mathrm{~h}$ UT, they do not refer to the 1392 dust trail. Rather, given the lower perihelion distance, we assume that they are due to even older trails. Our calculations put the age back at least 1000 years. Also, 15 orbits (35\%) have relatively low declination, corresponding to low orbital inclinations. This observation remains unaccounted for. It is not unlikely that other nearby resonances, such as the 11:13 resonance, may play a role, even though such higher resonances are narrower than the lower 6:7 resonance and have a smaller 
probability to trap particles. It is likely that future outbursts that belong to these other resonant windows will be identified, especially now that the comet approaches Earth's orbit.

\section{ACKNOWLEDGMENTS}

We thank observers of the California Meteor Society and the Dutch Meteor Society for support of the December 1994, 1997, and 2000 Ursid campaigns reported in this work. We thank all observers in Global-MS-Net that contributed data to this work. PJ acknowledges support from NASA's Planetary Atmospheres, Planetary Astronomy, and Exobiology programs.

\section{REFERENCES}

Asher, D. J., M. E. Bailey, and V. V. Emel'yanenko 1999. Resonant meteoroids from Comet Tempel-Tuttle in 1333: The cause of the unexpected Leonid outburst in 1998. Mon. Not. R. Astron. Soc. 304, L53-56.

Becvár, A. 1946. IAU Circ. 1026.

Borovicka, R., R. Stork, and J. Bocek 1999. First results from video spectroscopy of 1998 Leonid meteors. Meteorit. Planet. Sci. 34, 987-994.

Brown, P., and R. Arlt 2000. Detailed visual observations and modelling of the 1998 Leonid shower. Mon. Not. R. Astron. Soc. 319, 419-428.

Ceplecha, Z. 1951. Umids-Becvar's meteor stream. Bull. Astron. Inst. Czech. 2, $156-159$.

DeLignie, M., and C. Johannink 2001. Nederlandse videowaarnemingen van de Ursiden uitbarsting 2000. Radiant, J. Dutch Meteor Soc. 23, 71-75 (in Dutch).

Denning, W. F. 1912. Meteors on Christmas night Ursids. Observatory 35, 90-91.

Denning, W. F. 1921. Meteor notes, Dec. 1920. Observatory 44, 31-32.

Jenniskens, P. 1987. Ursiden 1986. Radiant, J. Dutch Meteor Soc. 10, 101-102 (in Dutch)

Jenniskens, P. 1988. Ursiden 1997. Radiant, J. Dutch Meteor Soc. 10, 21-22 (in Dutch).

Jenniskens, P. 1994. Meteor stream activity. I. The annual streams. Astron. Astrophys. 287, 990-1013.

Jenniskens, P. 1995. Meteor stream activity. II. Meteor outbursts. Astron. Astrophys. 295, 206-235.

Jenniskens, P. 1997. Meteor stream activity. IV. Meteor outbursts and the reflex motion of the Sun. Astron. Astrophys. 317, 953-961.

Jenniskens, P. 1998a. First results of Global-MS-Net: Annual report for 1997. WGN, J. IMO 26, 79-85.

Jenniskens, P. 1998b. On the dynamics of meteoroid streams. Earth Planets Space 50, 555-567.

Jenniskens, P. 2000a. Ursid meteors 2000. IAU Circ. 7544.

Jenniskens, P. 2000b. Ursid meteors 2000. IAU Circ. 7548.

Jenniskens, P. 2001a. Discoveries from observations and modeling of the 1998/99 Leonids. In Interplanetary Dust (E. Grün, B. A. S. Gustafson, S. F. Dermott, and H. Fechtig, Eds.), pp. 233-252. Springer-Verlag, Berlin.

Jenniskens, P. 2001b. Model of a one-revolution comet dust trail from Leonid outburst observations. WGN, J. IMO 29, 165-175.
Jenniskens, P., and H. Betlem 2000. Massive remnant of evolved cometary dust trail detected in the orbit of Halley-type Comet 55P/Tempel-Tuttle. Astrophys. J. 531, 1161-1167.

Jenniskens, P., and B. Å. S. Gustafson 2000. The rare 1932 dust trail encounter of 2000 November 17 as observed from aircraft. WGN, J. IMO 28, 209-211.

Jenniskens, P., and E. Lyytinen 2000. Possible Ursid outburst on December 22, 2000. WGN, J. IMO 28, 221-226.

Jenniskens, P., and E. Lyytinen 2001. 2000 Ursid outburst confirmed. WGN, J. IMO 29, 41-45.

Jenniskens, P., H. Betlem, M. de Lignie, and M. Langbroek 1997. The detection of a dust trail in the orbit of an Earth-threatening long-period comet. Astrophys. J. 479, 441-447.

Jenniskens, P., H. Betlem, M. de Lignie, C. ter Kuile, M. C. A. van Vliet, J. van't Leven, M. Koop, E. Morales, and T. Rice 1998. On the unusual activity of the Perseid meteor shower (1989-96) and the dust trail of Comet 109P/Swift-Tuttle. Mon. Not. R. Astron. Soc. 301, 941-954.

Jenniskens, P., C. Crawford, S. J. Butow, D. Nugent, M. Koop, D. Holman, J. Houston, K. Jobse, G. Kronk, and K. Beatty 2000a. Lorentz-shaped comet dust trail cross section from new hybrid visual and video meteor counting technique-implications for future Leonid storm encounters. Earth, Moon, Planets 82-83, 191-208.

Jenniskens, P., M. A. Wilson, D. Packan, C. O. Laux, C. H. Krüger, I. D. Boyd, O. P. Popova, and M. Fonda 2000b. Meteors: A delivery mechanism of organic matter to the early Earth. Earth, Moon, Planets 82-83, 57-70.

Kondrat'eva, E. D., and E. A. Reznikov 1985. Comet Tempel-Tuttle and the Leonid meteor swarm. Sol. Syst. Res. 31, 486-492.

Kresák, L. 1993. Cometary dust trails and meteor storms. Astron. Astrophys. 279, 646-660.

Langbroek, M. 1997. Conspicuous Ursid rates in 1996. Proceedings International Meteor Conference, poster presentation (IMO), Polsdam.

Lindblad, B. A., and D. I. Steel 1994. Meteoroid orbits available from the IAU Meteor Data Center. In Asteroids, Comets, Meteors 1993, Proceedings of the 160th International Astronomical Union, Belgirate, Italy, June 14-18, 1993 (A. Milani, M. Di Martino, and A. Cellino, Eds.). Int. Astron. Union Symp. 160, p. 497. Kluwer Academic, Dordrecht/Norwell, MA.

Lyytinen, E. J. 1999. Meteor predictions for the years 1999-2007 with the satellite model of comets. Meta Res. Bull. 8, 33-40.

Lyytinen, E. J., and T. Van Flandern 2000. Predicting the strength of Leonid outbursts. Earth, Moon, Planets 82-83, 149-166.

Lyytinen, E. J., M. Nissinen, and T. Van Flandern 2001. Improved 2001 Leonid storm predictions from a refined model. WGN, J. IMO 29, 110-118.

Marsden, B. G., and G. V. Williams 1994. Catalogue of Cometary Orbits, 9th ed. Smithsonian Astrophys. Obs., Minor Planet Center, Cambridge, MA.

McNaught, R. H., and D. J. Asher 1999. Leonid dust trails and meteor storms. WGN J. IMO 27, 85-102.

Ohtsuka, K. 1994. Expectation for enhancement of the Ursids activity in 1994. Tokyo Meteor Network Rep. 14, 85.

Ohtsuka, K., H. Shioi, and E. Hidaka 1995. Enhanced activity of the 1994 Ursids from Japan. WGN, J. IMO 23, 69-72.

Reznikov, E. A. 1983. Trudy Kazan Gor. Astron. Obs. 47, 131-136 (in Russian). Williams, I. P., and Z. Wu 1994. The current Perseid meteor shower. Mon. Not. R. Astron. Soc. 269, 524-528. 